\title{
Sulfasalazine Induced Toxic Epidermal Necrolysis in a Rheumatoid Arthritis Patient-A Case Report
}

\author{
Adhirai Raveendran ${ }^{1}$, Jayadev Betkerur², Sindhughata Ashok Archana², Adusumilli Pramod Kumar', \\ Madhan Ramesh ${ }^{1 *}$, Gurumurthy Parthasarathi ${ }^{1}$
}

1'Department of Pharmacy Practice, JSS College of Pharmacy, JSS University, Mysuru-570015, Karnataka, INDIA.

${ }^{2}$ Department of Dermatology, JSS Medical College and Hospital, JSS University, Mysuru-570004, Karnataka, INDIA.

\begin{abstract}
Toxic epidermal necrolysis also known as Lyell's syndrome is a rare but potentially life threatening severe cutaneous adverse reaction with widespread epidermal detachment and mucosal erosions. Drug exposure and a resulting hypersensitivity reaction is the cause of the very large majority of cases of Steven Johnson Syndrome. This is a case of 34-year-old female patient, presented to hospital with a presentation of rash, throat pain, fever and eye pain with redness. The patient medical history shows that she is a known case of rheumatoid arthritis and treated with sulfasalazine $500 \mathrm{mg}$, paracetamol $650 \mathrm{mg}$ and aceclofenac $100 \mathrm{mg}$ which she took for 16 days and injection methylprednisolone IM for 20 days. She has no improvement of joint pain and developed few fluid filled lesions in oral cavity, which ruptured spontaneously. She gives history of a pain and burning sensation while swallowing and her first biopsy report showed erythema multiforme on hospital admission and was finally diagnosed as Toxic Epidermal Necrolysis. Patient was treated with capsule cyclosporine and injection dexamethasone. The patient showed good response to treatment and lesions, erosions decreased within span of 20 days. Toxic epidermal necrolysis is a severe cutaneous adverse drug reactions associated with high mortality. Patients prescribed with sulfanamides like sulfasalazine, educating them regarding the appropriate use of medications are of utmost importance.
\end{abstract}

Key words: Toxic Epidermal Necrolysis, Severe Cutaneous Adverse Drug Reaction, Sulfasalazine.

\section{INTRODUCTION}

Toxic epidermal necrolysis (TEN) also known as Lyell's syndrome is a rare but potentially life threatening severe cutaneous adverse reaction (SCAR) with widespread epidermal detachment and mucosal erosions. Drug exposure and a resulting hypersensitivity reaction is the cause of the very large majority of cases of Steven Johnson Syndrome (SJS). ${ }^{1}$ Sulfasalazine is the most common drug prescribed for conditions like crohn's disease, ulcerative colitis, rheumatoid arthritis. Sulfasalazine causes many potential adverse reactions like headache, anorexia, nausea, vomiting, diarrhea, gastric distress, photosensitivity in more than $10 \%$ of the patients, SJS, pruritis, reversible oligospermia occur in less than 3\% and Sulfasalazine induced TEN is infrequent. TEN may be prove fatal if not treated promptly. ${ }^{2}$ The average reported mortality rate of TEN is $25-35 \%$. Herewith we are reporting a case of sulfasalazine induced TEN necessitating hospitalization.

\section{CASE REPORT}

A 34-year-old female patient is presented to tertiary care hospital with a presentation of rash since two days, throat pain since one day, fever and eye pain with redness since three days. The patient past history reveals that she has joint pain since 8 months and increased in the span of 4 months and was diagnosed with rheumatoid arthritis. Patient was started with sulfasalazine 500 $\mathrm{mg}$, paracetamol $650 \mathrm{mg}$ and aceclofenac $100 \mathrm{mg}$ which she took for 16 days and inj. Methylprednisolone IM for 20 days. She stopped medication for two days as pain reduced. She developed reddish fluid
Submitted date : $23 / 01 / 2016$ Accepted date : 16/02/2016

DOI: 10.5530/ijopp.9.1.11

Address for correspondence: Dr. Madhan Ramesh, Professor \& Head Department of Pharmacy Practice

JSS College of Pharmacy JSS University

Mysuru-570015, Karnataka, INDIA.

Phone no: +91-9901218640

E-mail: madhanramesh@ hotmail.com

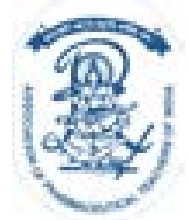

www.ijopp.org 


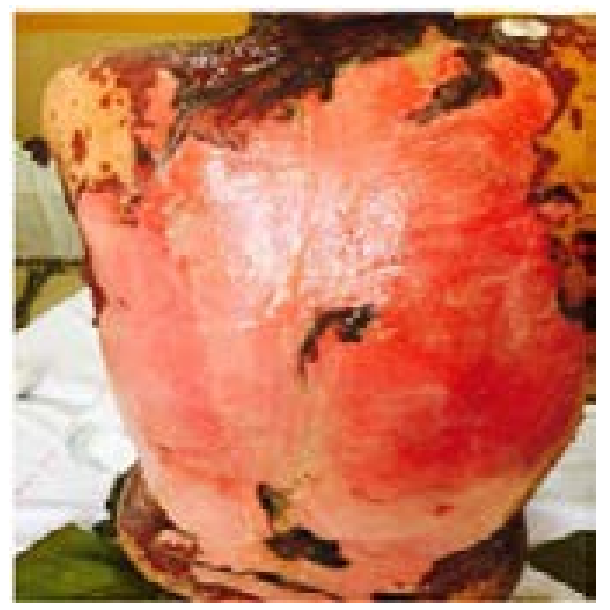

Figure 1: Patient presenting with TEN following sulfasalazine therapy

filled lesions admitted in hospital where she was given injection hydrocortisone $100 \mathrm{mg} \mathrm{IV}$, and injection dexamethasone $4 \mathrm{mg} I V$. She gives a history of no improvement and developed few fluid filled lesions in oral cavity, which ruptured spontaneously. She gives a history of pain and burning sensation while swallowing.

Cutaneous examination revealed multiple erythematous macules and target lesions (Figure 1) few with central vesicles and central petechiae, the forehead, nose, cheeks, chin, ears, neck, chest, abdomen, back, retroauricular areas and few discrete erythematous macules and papules, extensive peeling over the upper limbs and lower limbs. Nikolsky's sign was positive. Mucous membrane of eye, oral cavity and genitals were involved. Erosions, crusting and mucous discharge were present. Patient was treated with capsule cyclosporine $150 \mathrm{mg}$ for 14 days, $100 \mathrm{mg}$ for 6 days, $50 \mathrm{mg}$ for 4 days and stopped, injection dexamethasone $8 \mathrm{mg}$ for 7 days, $4 \mathrm{mg}$ for 5 days, $2 \mathrm{mg}$ for 3 days. The patient showed good response to treatment and lesions, erosions decreased within span of 20 days. The patient was discharged with complete recovery after 26 days.

Laboratory investigations was done after admission to the hospital, the reports were ESR $50 \mathrm{~mm} / \mathrm{hr}$, neutrophils $91.8 \%$, ALT $25 \mathrm{IU}, \mathrm{A} / \mathrm{G}$ ratio 1.0, albumin $3.3 \mathrm{~g} /$ dl. First Biopsy report showed erythema multiforme and second report was non specific.

\section{DISSCUSSION}

Toxic epidermal necrolysis is a known side effect of drugs commonly used in day to day practice. ${ }^{3}$ The various studies have shown that sulfamethoxazole/trimethoprim (SMX/TMP), sulfonamides (sulfasalazine, sulfadiazine, sulfadoxine, sulfafurazole), allopurinol, carbamazepine, phenytoin, phenobarbital and NSAID's of the oxicam-type (meloxicam, piroxicam, tenoxicam)

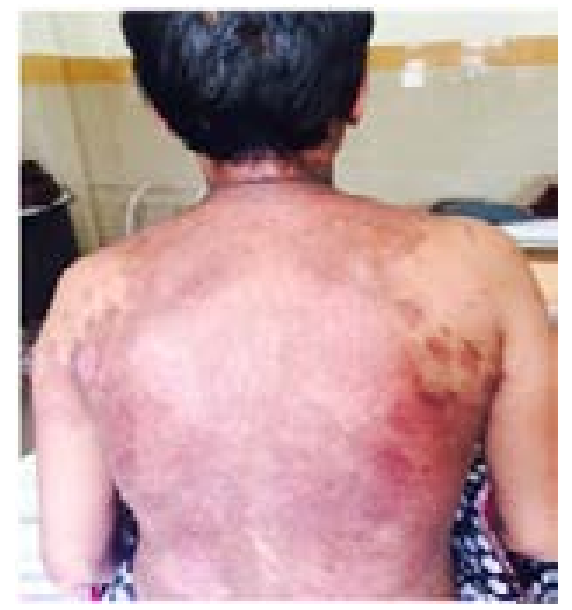

Figure 2: Patient recovered following drug withdrawal

are most common drugs implicated in the causation of TEN. ${ }^{1,5}$

Early recognition and discontinuation of responsible drug remains primary treatment of management in drug induced toxic epidermal necrolysis. The immediate treatment necessary depends upon the severity of reaction. Emergency care, maintaining fluid and electrolyte homeostasis, mitigating temperature loss, providing adequate analgesia, preventing secondary infection. Drugs like corticosteroids, antibiotics, antihistamines, anti-coagulant, antiseptic and analgesics can be used. ${ }^{1,4-6}$ In this case, the patient was treated with immunosuppressant and corticosteroids. The role of immunosuppressant in this patient is to reduce the progression of disease. When compared to steroids, cyclosporine has a greater benefit in TEN patients. Cyclosporine (cyclosporin $\mathrm{A}, \mathrm{Cs} \mathrm{A}$ ) acts by blocking the transcription of cytokinegenes in activated $\mathrm{T}$ cells. ${ }^{7} \mathrm{CsA}$ forms a complex with cyclophilin inhibit the phosphatase activity of calcineurin, which regulates nuclear translocation and subsequent activation of nuclear factor of activated T-cells (NFAT) transcription factors. In addition to the calcineurin/NFAT pathway, recent studies indicate that CsA also blocks the activation of Jun amino terminal kinase (JNK) and p38 signaling pathways triggered by antigen recognition, making CsA a highly specific inhibitor of T cell activation. ${ }^{8,9}$

In TEN apoptosis of epithelial cells was principally mediated through the activation of the Fas (CD95) receptor, and those commercial preparations of human immunoglobulins contained variable amounts of Fasblocking anti-bodies capable of inhibiting the activation of the death receptor.

The role of corticosteroids has been controversial for many years in TEN. Steroids have been accepted as a treatment option as they suppress the necrolytic pro- 
cess in the skin and internal organs. A study shows that early treatment with corticosteroids reduced morbidity and improved survival in patients of SJS and TEN. Studies have also reported better outcome in patients treated with methyl prednisolone and dexamethasone pulse therapy. Literature search has also revealed that corticosteroids may prove beneficial in cases of TEN due to their antiapoptotic effect on keratinocytes. ${ }^{10}$

After treatment with corticosteroids and cyclosporine, reaction subsided within 3 weeks time (Figure 2). It is very essential that patients should be informed regarding the offending agent so as to avoid future complications associated with drug use.

\section{CONCLUSION}

Toxic epidermal necrolysis is a severe cutaneous adverse drug reaction (SCAR) associated with high mortality. Patients prescribed with sulfanamides like sulfasalazine, educating them regarding the appropriate use of medications are of utmost importance. It is also advisable to provide personalized "alert card", with the description of adverse drug reaction, to the patient who suffered from such serious reactions. The patient recovered from the reaction after the specific and symptomatic treatment of the presenting illness.

\section{ACKNOWLEDGEMENT}

Authors acknowledge the Pharmacovigilance Programme of India, Indian Pharmacopoeia Commission for the support.

\section{CONFLICTS OF INTEREST}

The author have no conflict of interest.

\section{ABBREVIATION USED}

JNK: Jun amino terminal kinase

NFAT: $\quad$ Nuclear Factor of Activated T-cells

SCAR: $\quad$ Severe Cutaneous Adverse Drug

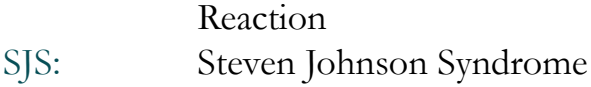

SMX/TMP: Sulfamethoxazol/ trimethoprim

TEN: $\quad$ Toxic Epidermal Necrolysis

\section{REFERENCES}

1. Harr T, French LE. Toxic Epidermal Necrolysis and Stevens-Johnson Syndrome. Orphanet Journal of Rare Disease. 2010;5(1):39.

2. McEvoy KG, Snow KE, Kester LMS, Kathy LMS. American society of health system pharmacists. Authority of American Society of Health System Pharmacists. 2005;1(1):396-9.

3. Kimble MAK, Young LY. Applied therapeutics: The clinical use of drugs. 9:38 16.

4. Saha K. Toxic Epidermal Necrolysis: Current concepts in pathogenesis and treatment. Indian Journal of Dermatology Venereology and Leprology. 2000;66(1):10.

5. Qadir SNR, Raza N, Qadir F. Drug induced toxic epidermal necrolysis. Journal of Medical Case Reports. 2009;2:1-5.

6. Kumar MM, Yogendra, Karthik, Gowthami B, Yogananda R. Toxic Epidermal Necrolysis induced by phenytoin: A case study. Indian Journal of Pharmacy Practice. 2014;7(2):37-9.

7. Toxic Epidermal Necrolysis. Medscape.http://emedicine.medscape.com/ article/229698-overview(Accessed on 29 Aug 2015).

8. Pise HN, Padwal SL. Diclofenac induced Angioedema: A case report. Asian Journal of Pharmaceutical and Clinical Research. 2015;8(2):4-5.

9. Arévalo JM, Lorente JA, González-Herrada C, Jiménez-Reyes J. Treatment of toxic epidermal necrolysis with cyclosporin A. The Journal of Trauma. 2000; 48(3):473-8.

10. Azfar NA, Zia MA, Malik LM, Khan AR, Jahangir M. Role of systemic steroids in the outcome of Stevens-Johnson syndrome and toxic epidermal necrolysis. Journal of Pakistan Association of Dermatologists. 2010;20:158-62. 